\title{
Abstracts of the Contributions Second Scientific Congress of the African Association of Veterinary Anatomists Cairo, March 20-21 2010
}

\section{Nasal and Paranasal Sinuses of the Donkey: Gross Anatomy and Computed Tomography}

\author{
Samir A.A. Elgendy and Mohamed A.M. Alsafy \\ Anatomy and Embryology Dept. Fac. Vet. Med. Alex. Univ. \\ E-mail:elgendyanatomist@yahoo.com
}

\begin{abstract}
The current work aimed to analyse the anatomical findings of the nasal and paranasal sinuses of the donkey by gross anatomical observation of the skull and cadaver head, cross-sections, sagittal sections and computed tomography (CT) imaging. Seven adult donkeys and ten skulls were used in this study. The donkey head had six pairs of sinuses, three paranasal; the frontal, maxillary, and sphenopalatine sinuses and three nasal; dorsal, middle, and ventral conchal sinuses. The left and right frontal sinuses were completely divided by a thick bony septum. Each frontal sinus is united rostrally with the dorsal conchal sinus forming conchofrontal sinus that communicated directly with the caudal maxillarysinus by frontomaxillary opening. The maxillary sinus is divided into rostral and caudal compartments by a thin incomplete bony septum. The caudal maxillary sinus is divided into medial and lateral compartments by the infraorbital canal that run longitudinally through it. The rostral maxillary sinus is communicated with the ventral conchal sinus through the conchomaxillary opening, while the rostral and caudal maxillary sinuses are connected with the middle nasal meatus via the slit-like nasomaxillary opening. Approach to the interior of the frontal, rostral and caudal maxillary was determined. The location, size, and shape of the paranasal sinuses openings (conchomaxillary, nasomaxillary, frontomaxillary and sphenopalatinal openings and opening of middle conchal sinus into caudal maxillary sinus) communicated the sinuses with the nasal cavity were identified in CT. The frontomaxillary opening was the largest opening, while the nasomaxillary opening was the narrowest one. CT images with cross-sections provided clear, unobstructed images on the paranasal structures, while paranasal lining was unclear in CT images because of its thin layer.
\end{abstract}

\section{Prenatal Development of the Adrenal Gland in the One Humped Camel (Camelus dromedarius).}


El-Nahla, Sanaa. M; Imam, H.M; M; Moussa, E.A and Elsayed, A.K. (2009)

Department of Anatomy \& Embryology, Faculty of Veterinary Medicine, Ismailia - Suez Canal University

\begin{abstract}
The current study have been achieved to elucidate the prenatal development of the adrenal gland throughout the whole fetal life in the one-humped camel; regarding its microscopic description of the cellular components of both cortex and medulla, aiming to give adequate information about the histogenesis and morphogenesis in such valuable and unique animal.

The material comprised the adrenal glands of 100 camel embryos and fetuses, collected from El-Basateen and El-Qureen slaughterhouses (70 for ordinary stains and 30 for special stains).
\end{abstract}

The specimens for ordinary histological stains were preserved in $10 \%$ neutral buffered formalin while those for special stain of chromaffin reaction were preserved in solution contain potassium dichromate.

The study of the onset and development of adrenal cortex and medulla were examined carefully. The differentiation of the cortical zones and development of the adrenal capsule were thoroughly investigated.

Diagrams were also made and the results described in details and discussed with available literatures on the camel, other animals and birds.

\title{
Localization of Transforming Growth Factor ß1 (TGF ß1) on the Testis of Brown Banded-Bamboo Shark (Chiloscyllium punctatum)
}

\author{
Kassab M'. and Yanai $\mathbf{T}^{2}$ \\ 1-Department of Cytology and Histology, Fac. Vet. Med., Kafr el-sheikh Univ., Egypt. \\ 2-Department of Pathology, Fac. of Biological Science, Gifu Univ., Japan.
}

\begin{abstract}
Growth factors are shown to promote cell proliferation, regulate tissue differentiation, and modulate organogenesis. In the present investigation, the Localization of transforming growth factor B1 (TGF ß1) was studied on the testis of brown bandedbamboo shark by immunohistochemistry. The immunohistochemical reaction is present in the somatic cells mainly Leydig cells in most of zones in the summer season, while in
\end{abstract}


the winter season the expression is confined to the Leydig cells of the spermatocytes, spermatids and spermatozoal zones. The reaction in the germ cells is clear in the spermatogonia of the germinal zone in the summer season and the head of sperms in the spermatozoal zone in the winter season and negative in other zones in the two seasons. In conclusion, such findings may suggest that TGF $B 1$ is important paracrine and/or autocrine regulators of spermatogenesis through its direct effect on steriodogenesis either stimulate or inhibit activity of Leydig cells.

\title{
Comparative Anatomical Study on the Vomeronasal Organ in Sheep and Dog
}

\section{Gamal Aloush}

Faculty of Veterinary Medicine, Al-Baath University,Hamah, Syria

\begin{abstract}
The study shows that there are clear anatomical differences in the vomeronasal organ in both sheep and dog.

In both animals the vomeronasal organ occupies the same position in the cranial part of the nasal cavity and has the same relation. It receives blood supply from the sphenopalatine and caudal palatine arteries.

In sheep, the vomeronasal organ is $U$ - shaped and opens dorsolaterally. Its length is about $6 \mathrm{~cm}$ and it has different diameters along its course and has small and narrow cavity.

In dog, the vomeronasal organ is clearly developed and is $\mathrm{J}$-shaped. Its length is about $12 \mathrm{~cm}$ and has a uniform diameter along its course. It is divided into three parts and has a large and long cavity which ends at the level of third molar tooth.
\end{abstract}

\section{The Effect of Thermal Manipulation during Embryogenesis of Broiler Chicks on Thyroid Hormones and Blood Parameters in Post Hatch Chick}

\footnotetext{
Alzghoul, M. B ${ }^{1}$., Jawasreh K. I ${ }^{3}$,, Al-Rukiabat, R. K. ${ }^{2}$, Abu Basha E' . Department of Basic Medical Veterinary Science ${ }^{1}$, Department of animal Health and pathology ${ }^{2}$. Jordan University of Science and Technology. National Center for Agriculture and Extension (NCARE)
} 


\begin{abstract}
Thermal manipulation, in which the embryos are exposed to high or low temperature during certain periods of broiler embryogenesis, has been addressed as a technique to enhance body weight and to improve thermotolerance acquisition of broiler chickens.

This study was conducted to elucidate the effect of thermal manipulation at $38.5^{\circ} \mathrm{C}$ and $40^{\circ} \mathrm{C}$ for 3,6 and 24 hour during late embryogenesis (E16-E18) on broiler chick's body weight and thermotolerance acquisition post hatching.

Four hundred and fifty (450) eggs were divided into three groups of 150 each. One group was considered as control group and eggs were incubated at $37.8^{\circ} \mathrm{C}$ and $56 \%$ relative humidity $(\mathrm{RH})$. The second group was thermally manipulated during late embryogenesis embryonic day $(\mathrm{E} 16-\mathrm{E} 18)$ at $38.5^{\circ} \mathrm{C}$ and $65 \%$ relative humidity $(\mathrm{RH})$ for period of 3,6 and 24 hour. The third group was thermally manipulated during late embryogenesis as group two except changing the temperature from $38.5^{\circ} \mathrm{C}$ to $40^{\circ} \mathrm{C}$.
\end{abstract}

During the chick's life span from day 1 to day 42, body weight and body temperature were recorded. In addition, these parameters were measured at thermal challenge (TC) days $(14,28$ and 42). Blood samples were collected at thermal challenge days for measuring T3, T4 and IGF-I levels in the serum and other blood parameters evaluation (PCV, TWBC counts, differential leukocytes counts, and H/L ratio).

The serum levels of thyroid hormone T4 were higher in thermal manipulated chicks at all days of TC as compared to controls while the serum levels of T3 were lower in thermal manipulated chicks as compared to controls. In addition, the serum IGF-I levels in thermal manipulated chicks was higher than control at days 28 and 42 of TC but lower than control at the age of 14 day. Furthermore, there were significant changes in the blood parameters (PCV, TWBC, differential leukocytes counts, and H/L ratio) between the treated chicks and controls. The PCV and TWBC results related to the manipulation at $38.5^{\circ} \mathrm{C}$ shows that treated chicks are resistant to heat stress and this will improve thermo tolerance acquisition. While the packed cell volume for thermal manipulated chicks at $40^{\circ} \mathrm{C}$ was not different from the control chicks. On the other hand, TM resulted in reduced the TWBC counts for thermal manipulated chicks at $40^{\circ} \mathrm{C}$ as compared to controls. In addition, the differential leukocyte counts and $\mathrm{H} / \mathrm{L}$ ratio results at the age of 28 and 42 day gives indication about thermally manipulated chicks. They were resistant to heat stress at these ages.

It can be concluded that TM for 3, 6 and 24 hour during late embryogenesis (E16-E18) resulted in improved chickens performance and also resulted in enhanced body weight at the marketing age (D42) especially the manipulation at $40^{\circ} \mathrm{C}$. Furthermore, TM resulted in reduced T3 hormone levels without effect on $\mathrm{Tb}$. Observations related to blood parameters are indication of improving thermo tolerance acquisition. 


\title{
Histomorphometric Studies of the Urinary Tubules of the African Grasscutter (Thryonomys swinderianus).
}

\author{
${ }^{* 1} I . E$. AJAYI, ${ }^{2}$ S. A. OJO, ${ }^{3}$ J. O. AYO and ${ }^{2}$ C. S. IBE \\ ${ }^{1}$ Department of Veterinary Anatomy, University of Abuja, Abuja, Nigeria. \\ ${ }^{2}$ Department of Veterinary Anatomy, Ahmadu Bello University, Zaria, Nigeria. \\ ${ }^{3}$ Department of Veterinary Physiology and Pharmacology, Ahmadu Bello University, Zaria, Nigeria.
}

\begin{abstract}
The study was carried out to elucidate special features in the structure of the urinary tubules in the grasscutter (Thryonomys swinderianus). Seven adult, apparently, healthy grasscutters were used in this study. They were anaesthesized with chloroform, dissected and the kidneys removed. The kidneys were fixed and processed by routine histological procedures. The various segments of the nephron were identified by their cell types and their diameters were measured. The data obtained were subjected to Pearson's correlation analysis. The diameters of the Bowman's capsule and glomerulus were $11.24 \pm 0.460$ and $9.21 \pm 0.347 \mu \mathrm{m}$, respectively. There was a very strong positive correlation between the diameters of the Bowman's capsule and those of the glomerulus $(r=0.975, P<0.01)$. A significant positive correlation was also observed between the distal convoluted tubule and the thin segment of the loop of Henle $(r=0.786, P<0.05)$, the collecting duct and the proximal convoluted tubule $(r=0.781, P<0.05)$, and between the collecting duct and the thin segment of the loop of Henle $(r=0.821, P<$ 0.05 ). This study has attempted to present base-line data of the diameters of the various segments of the nephron.
\end{abstract}

\section{Reconstitution of the anatomy museum to facilitate integrated self-directed learning}

\author{
El-Khaligi, G. M.; Wally. Y. R. and A. R. Tolba \\ Department of Anatomy\&Embryology, Faculty of Vet, Med, Cairo University
}

\begin{abstract}
In the last decade the nature of the anatomy education has changed due to both a new kind of students who learn differently from those of past years and the advances in the anatomical imaging and viewing. In recent years, many anatomists have recognized the importance of entrance of technologies in facilitating the delivery of anatomical information through the museum (Lamperti and Sodicoff 1997; Carmichael and Pawlina 2000; Trelease 2002; and Venuti, Imielinska and Molholt. 2004). The value
\end{abstract}


of introducing an assisted instruction into the traditional anatomy curriculum in an effort to reduce the time and cost associated with cadaver dissection.

This work was conducted in two parts. Part I: the reconstituting and rearrangement of the anatomy museum faculty of veterinary medicine Cairo University in the form of modular center in which its basic unit was the module. So we prepare different learning materials including anatomical dissected specimens, museum-jars specimens, plastinated specimens, colored bones, casts, models, radiographs, photos used by hand, atlases and charts in addition to many paper and computer assisted recourses as handouts, books, frequent quizzes for self assessment by the students, short videos, electronic books, needed sites and CDs contain lectures prepared in power point presentations for anatomy, histology, pathology, surgery, medicine and pharmacology to the specific topic used in the module. Part II: A descriptive survey applied on 592 veterinary educators all over Arab republic of Egypt to analyzed ten aspects of comparison between self learning modules using multimedia as a method of teaching with the traditional learning. The results of the survey revealed that, the students recommended its use with a record $95.27 \%$ and other students not recommended with a record $4.72 \%$. The results obtained were discussed with those concerned with the use of integrated self directed learning.

\title{
Testicular Morphometry and Stages of Spermatogenic Cycle in the Greater Cane Rat (Thryonomys swinderianus Temminck)
}

\author{
${ }^{*}$ Adebayo, A. O. ${ }^{1}$, Akinloye, A. $\mathrm{K}^{1}$, Olurode, S. $\mathrm{O}^{2}$ \\ ${ }_{1}^{1}$ Department of Veterinary Anatomy, University of Agriculture, Abeokuta. \\ ${ }^{2}$ Department of Veterinary Public Health and Reproduction, University of Agriculture, Abeokuta.
}

\begin{abstract}
The testis of the greater cane rat, a wild hystricomorphic rodent found only in Africa and currently undergoing domestication in the West African sub region, was investigated in this work. The gross morphometry of the testis in this animal showed a mean testicular length, width and volume of $1.95 \pm 0.22 \mathrm{~cm}, 1.09 \pm 0.16 \mathrm{~cm}$ and $1.25 \pm 0.45 \mathrm{ml}$ respectively. The mean weight of the testis was $1.18 \pm 0.32 \mathrm{~g}$ while a low relative testicular weight of $0.053 \%$ was observed. The histology of the testis revealed a normally formed germinal epithelium that contained all cellular stages of spermatogenesis in the seminiferous tubules. Based on the changes in the nuclear morphology and development of the acrosomic system in the transformation of spermatids to spermatozoa, nine (9) stages of the spermatogenic cycle were recognized and characterized in this animal. The seminiferous tubule had a mean diameter of $169.64 \pm 13.88 \mu \mathrm{m}$ with epithelial height of $42.85 \pm 4.05 \mu \mathrm{m}$. The quantitative and qualitative data obtained in this work provide basis for further studies on the reproductive biology as well as in assessing the effects of
\end{abstract}


certain pathological and pharmacological conditions on the testis of this animal. The stages of the spermatogenic cycle characterized in this work can aid in the determination of the spermatogenic efficiency which is a valuable tool towards increasing stock levels and intensification of production practices of the cane rat.

\title{
Peculiar Anatomical Features of Dromedary vis-à-vis Surgical Procedures
}

\section{T.K.Gahlot}

Department of Veterinary Surgery and Radiology. College of Veterinary and Animal Science Bikaner, Rajasthan, India

\begin{abstract}
Dromedary camels have peculiar anatomy of many organs which makes it different from other animals from surgery point of view. A distensible out pouching of soft palate of camel is not seen in other species. Injury to soft palate leads to either its trapping 'in situ' or it hangs outside oral cavity. Its resection is done close to its attachment and needs per-oral approach for surgery. Mandibular fracture occurs commonly in male camels because of powerful jaw muscle action on a weak mandible. Long inter-dental space, presence of alveoli of first premolar or tushes and presence of mental canal make the lower jaw weak and fracture occurs at this point. Camels prehense with lips and when they go apart after fracture, they are unable to prehense. Camels dip half of the mouth for drinking water from bucket or any such source. Application of plaster of Paris or internal fixation techniques donot prove successful to immobilize these fractures. Interdental wiring proves unique, economical and effective technique for immobilization of mandibular fractures in camels. Wires are passed through spaces between first two check tooth and central incisors and knots are applied. Such an anatomical advantage is not seen in other species to treat similar fractures.
\end{abstract}

Urethrostomy is done prescrotally in camels in cases of obstructive urolithiasis because of prescrotal location of sigmoid flexure. Testicles are situated high in perineal region, thus are predisposed to camel bites and provide excellent drainage of open castration wounds. Chest pad wounds are often soiled and contaminated because these touch ground during sternal recumbency, therefore need to be covered for protection during healing period. Similar protection is provided to the foot pad in cases of punctured foot. Anatomical presence of digital cushion needs special management to prevent its protrusion in deep punctured foot cases. Saddle gall wounds occur commonly in camels and surgical debridement and drainage are provided. Necrosis of skin and thoracic muscles occur in these cases. Present paper describes such surgical anatomy features in light of various types of surgeries. 


\title{
Light and Scanning Electron Microscopical Study on the Oviduct of the Ostrich (Struthio camelus)
}

\author{
A.S, Saber*, S.A.M. Emara*, O.M.M. AboSaeda** \\ * Faculty of Veterinary Medicine, Sadat City Branch, Menoufiya University, EGYPT \\ ** Hospital of Fac. Vet. Med., Sadat City Branch, Menoufiya University, EGYPT
}

\begin{abstract}
Nine mature female ostriches (Struthio camelus) aged 37- 49 months were used for the present study. The samples were taken during the period from September to November from Al-masreya ostrich farm, immediately after scarification and evisceration. Pieces from the different segments of the left oviduct were then fixed in $10 \%$ neutral buffered formalin and Bouin's and Susa solutions for the light and in $2 \%$ gluteraldehyde for the scanning electron microscopic studies. In addition, some samples were also processed for transmission E/M examination (Joel JEM 100 CXII).
\end{abstract}

For light microscopic study the slides were stained with Harri's hematoxylene and eosin, Crossmon's trichrome stain, Weigert's elastic tissue stain and Periodic acid Schiff technique (PAS).

The right oviduct is rudimentary, while the left one is well developed and is formed of five segments, namely: infundibulum, magnum, isthmus, uterus, and vagina.

The scanning electron microscope revealed that the lining epithelium of oviduct is folded and formed of pseudostratified columnar ciliated cells. The cilia were slender, dense uniformly arranged, and identical in length.

In magnum, isthmus and uterus the scanning E/M study revealed the presence of openings between the cilia for the secretion of the tubular glands of the lamina propria.

The results obtained were discussed with the available literatures in other bird species.

\section{Computed Tomography and 3D Reconstruction of the Respiratory Organs of the Egyptian Tortoise (Testudo kleinmanni)}

\author{
A.S. Saber and Basma M. Kamal \\ Department of Anatomy and Embryology, Faculty of Veterinary Medicine, Sadat city Branch, \\ Menoufiya Univcersity
}

\begin{abstract}
The study is designed to identify, describe and illustrates the topography of the respiratory organs of the Egyptian tortoise (Testudo kleinmanni) by using the digital camera and computed tomography. This may aid to obtaining a complete data on
\end{abstract}


dissection procedure, the topography and the initial sections outline and the safety of access in surgical procedures. The core of present study is a series of photographs showing stages of the dissection. A detailed description of the dissection method and anatomy of the exposed organs were also given. Thirteen wild adult live tortoises, Testudo kleinmanni, (three males and ten females) were collected from Marsa Matroh and Cairo governorates with an age ranging from 15-19 years, weight from 430-450 $\mathrm{gm}$, and a carapace, plastron length $10.5-11 \mathrm{~cm}$ and $9.5-9.8 \mathrm{~cm}$ respectively. Computed tomography provides detailed information on the respiratory system, the location of the coelomic structures with respect to the carapace (the upper shell) and the vertebrae, and to compare cross-sectional anatomic specimens that obtained by the digital camera with images obtained via computed tomography sections of the coelomic structures.

\title{
Spatiotemporal Distribution of Chondroitin Sulfate Proteoglycans in Developing Mice Retina and Optic Nerve
}

\author{
Safwat Ali Mohamed ALI), 2), Yoshinao Z. HOSAKA ${ }^{1), 2)}$, Masato \\ UEHARA ${ }^{1), 2)}$ \\ 1) The United Graduate School of Veterinary Science, Yamaguchi University, Yamaguchi 753- \\ 8515 \\ ${ }^{2)}$ Department of Veterinary Anatomy, Tottori University, Tottori 680-8553, Japan
}

\begin{abstract}
The aim of the present study was to determine the presence and distribution of proteoglycans in the mouse retina and optic nerve during embryonic, early postnatal and adult stages by immunohistochemistry using specific antibodies. No immunostaining for keratan sulfate was detected either in the retina or optic nerve from embryonic day (E) 12 to postnatal day (P) 120. At E12, no immunostaining was detected for chondroitin sulfate proteoglycans (CSPGs) in the retina. At E18, chondroitin-4-sulfate (C4S), chondroitin-6-sulfate (C6S) and biglycan were detected in the retina and optic nerve. However, staining for aggrecan was seen in the retina but not in the optic nerve. At P7, aggrecan immunostaining and biglycan immunostaining were clearly observed in the optic nerve, inner nuclear layer and ganglion cell layer and diffuse in the outer retina. C4S showed diffuse distribution in the retina and optic nerve, but C6S staining was mainly confined to the photoreceptor layer and optic nerve sheath. At P42, when the retinal layers had fully differentiated, biglycan showed diffuse distribution in the retina and optic nerve with intense staining in nerve-fiber rich layers but did not show immunostaining in the photoreceptor layer. Aggrecan showed no immunostaining in the photoreceptor layer and weak staining at the inner plexiform layer with higher density in the outer and inner nuclear layers, outer plexiform layer and ganglion cell layer. Both C4S and C6S were detected in the optic nerve and retina, but C4S was not detected at the photoreceptor layer and C6S showed strong immunostaining in the photoreceptor layer. The distributional pattern of CSPGs in P120 mouse retina and optic nerve was
\end{abstract}


similar to that in P42 mouse retina. The differences in distribution of these proteoglycans with respect of time course and cellular localization during development of the retina and optic nerve suggest that they may have unique or overlapping roles in development and maintenance of the retina and optic nerve.

\title{
Anatomical and Biochemical Studies on the Otolith of Telapia nilotica
}

\section{Omar,A.; Mervat Konsowa,M.H.;Helal,A.}

Department of Anatomy and Embryology.Faculty of Veterinary Medicine.Zagazig University.Egypt E-mail: mkonsowa @zu.edu.eg

\begin{abstract}
One-hundred otolithic crystals were extracted from 50 heads of River Nile Telapia nilotica. They were thoroughly washed for morphological studies, and exposed for biochemical analysis. Also, their crystal particles were examined microscopically, to confirm their chemical composition according to the shape of their specules. The results revealed that, the otolith appeared as oval milky, white stone-like crystals. Each one was lodged in a deep fossa at the floor of the cranial cavity, just lower to the brain. The otolith crystals were not bony in their structure or texture, where they formed mainly from calcium carbonate matrix. They also do not contain any collagenic fibers. The otolith (ear - stone) had a good role in the process of equilibrium and balance of the fishes. This research was carried out to fulfill the topographic anatomy of the otolith of Telapia nilotica as a main part of its internal ear.
\end{abstract}

\section{Some Comparative Immunohistochemical Studies on the Ductus Deferens in the Donkey (Equus Asinus) and Water Buffalo Bull (Bubalus bubalis).}

\author{
Mohamed Alkafafy'), Hossam Attia ${ }^{2)}$, Reda Rashed ${ }^{3)}$, Mohamed \\ Kandiel $^{4)}$ \\ 1) Department of Cytology and Histology, Faculty of Veterinary Medicine, Minoufiya University, \\ Sadat City Branch, Egypt. \\ 2) Department of Cytology and Histology, Faculty of Veterinary Medicine, Benha University, \\ Tokheh, Kaliobia, Egypt. \\ ${ }^{3)}$ Department of Anatomy and Embryology, Faculty of veterinary Medicine, Minoufiya University, \\ Sadat City branch, Egypt. \\ ${ }^{4)}$ Department of Theriogenology, Faculty of Veterinary Medicine, Benha University, Tokheh, \\ Kaliobia, Egypt.
}




\begin{abstract}
The current work aimed to apply immunohistochemistry (IHC) to evaluate the differential distribution pattern of some biologically active proteins in the ductus deferens (DD) in two different animal species common in the Egyptian farms. Immunolocalization of S100, angiotensin converting enzyme (ACE), $\alpha$ - smooth muscle actin ( $\alpha$-SMA), connexin 43 (Cx43) and galactosyltransferase (GalTase) was practiced on paraffin-embedded sections of DD from clinically healthy adult five donkeys and five water buffalo bulls. The $\mathrm{DD}$, in both species, was lined by a folded, high cuboidal (in donkey) or tall columnar (in buffalo) pseudostratified epithelium with stereocilia. IHC revealed that all antibodies used, displayed variable reactivities in the two species. Nerves and the periductal smooth muscles expressed a strong immunoreactivity (IR) for S100 and $\alpha-S M A$ respectively; both in the donkey and in the buffalo. Stereocilia of principal cells showed a marked ACE-immunostaining in the donkey but not in the buffalo. Cx43-binding sites were found between the basal portions of the ductal epithelium and the subjacent interstitium; as well as among the periductal smooth muscle cells (SMCs) only in the buffalo bulls. Distinct GalTase-binding sites were found in the ductal epithelium only in the donkey. In conclusion the present findings exhibited a species-specific pattern of distribution for most of proteins under study, suggesting a variable functional significance of the DD in the different animal species.
\end{abstract}

\title{
Prenatal Development of the Pars Intermedia of the Pituitary Gland in the Water Buffalo (Bubalus bubalis).
}

\author{
Hossam Attia ${ }^{a,}$, , Mohamed Alkafafy, ${ }^{\text {b) }}$, Reda Rashed ${ }^{\text {c) }}$ \\ a) Department of Histology and Cytology, Faculty of Veterinary Medicine, Benha University, Egypt \\ b) Department of Histology and Cytology, Faculty of Veterinary Medicine, Minuofiya University, \\ Sadat City Branch, Egypt \\ c) Department of Anatomy and Embryology, Faculty of Veterinary Medicine, Minuofiya University, \\ Sadat City Branch, Egypt
}

\begin{abstract}
This study was carried out on pituitary glands collected from buffalo embryos and fetuses with a crown vertebral rump length (CVRL) ranging from 1.5 to $95 \mathrm{~cm}$. The specimens were immediately immersed in the fixatives at least 3 days and paraffin embedded for routine histological work. The primordia of the pars intermedia (PI) appeared at $1.5 \mathrm{~cm} \mathrm{CVRL}$ as group of undifferentiated mesenchymal cellular band. This band was completely enclosing the pars nervosa (PN) at $3.2 \mathrm{~cm}$ CVRL. It appeared thick ventrally around the bottom of the PN. It was separated from the pars distalis (PD) via the hypophyseal cleft and from the neural lobe via thick reticular connective tissue fibers. These cells were differentiated to chromophils and chromophobes at $24 \mathrm{~cm}$ CVRL. The chromophils were exclusively basophils. Melanotrophs were represented by polyhedral cells, seen among the follicular cells at $35 \mathrm{~cm}$ CVRL. Adrenocorticotrophs appeared at $75 \mathrm{~cm}$ CVRL.
\end{abstract}




\title{
Immunohistochemical Distribution of Some Regulatory Peptides in the Rat Stomach
}

\author{
Elnasharty, $\mathbf{M}^{1}$ and Alkafafy, $\mathbf{M}^{2}$ \\ ${ }^{1}$ Department of Histology and Cytology, Faculty of Veterinary Medicine, Alexandria University \\ (Damanhur Branch), Elbostan, Beheira, Egypt \\ ${ }^{2}$ Department of Cytology and Histology, Faculty of Veterinary Medicine, Minuofiya University, \\ Sadat City Branch, Egypt
}

\begin{abstract}
The innervation of the rat stomach has been re-analyzed using cholinergic markers including peripheral (pChAT) and common (cChAT) types of choline acetyltransferase which is the product of a splice variant of ChAT mRNA and preferentially localized to peripheral cholinergic nerves and vesicular acetylcholine transporter (VAChT) as well as with nitric oxide synthase (NOS1) and tyrosine hydroxylase (TH). Paraffin embedded tangential section from the rat stomach were immunostained and used in the current work.

In the present study, we demonstrated accepted cholinergic and nitrergic neurons and nerve fibers in the rat stomach using antibodies to PChAT, cChAT and NOS1. Ganglionated submucosal plexus was almost absent from the gastric wall except for some scattered neurons. Most myenteric ganglionic neurons in the stomach were immunostained for pChAT, cChAT and NOS1 only whereas VAChT- and TH-immunoreactivities were observed in the form of varicose nerve fibers and nerve terminals in rat gastric wall.
\end{abstract}

These results indicate that the gastric wall nerve fibers expressed, heterogeneously, all the examined regulatory peptides while the myenteric neurons expressed only pChAT, cChAT and NOS1, suggesting a morphological evidence for the significant roles of cholinergic, nitrergic and adrenergic mechanisms in stomach secromotor functions

\section{The Lamination and Arterial Blood Supply of the Masseter Muscle of Camel (Camelus dromedarius)}

\author{
E.F. Khalifa and S.M. Daghash \\ Department of Anatomy \& Embryology, Faculty of Veterinary Medicine, Cairo University
}

\begin{abstract}
In the Herbivorous mammals, the Masseter muscle is the largest muscle of mastication for grinding solid plants (Smith and Savage, 1959; Turnbull, 1970; koppe et al., 1987).


The lamination of the Masseter muscle was examined in several herbivorous mammals (e.g. Yoshikawa et al., 1961, 1962; Sasali et al., 2001; Daghash, 2007; Wally et al., 2008). However, little is known about the structure and arterial blood supply of the Masseter muscle of the Camel. So, this study was conducted to give detailed information to increase the anatomical knowledge for the animal which may be of help in clinical work. The Masseter muscle of the camel was recognized into eight layers; first, second and third superficial layers, rostral deep layer, first and second caudal deep layers, Maxillomandibularis layer and Zygomaticomandibularis layer. The arterial blood supply was mainly through several masseteric branches originated from Transverse facial, Superficial temporal, Buccal and Mandibular labial arteries.

\title{
The Arterial Blood Supply of the Lung of the Donkey (Equus asinus).
}

\author{
F.M. Farag and E.F.Khalifa \\ Department of Anatomy and Embryology, Faculty of Vet. Med. Cairo Univ.
}

\begin{abstract}
The pulmonary vasculature of the lung was studied on twenty lungs of donkeys of different ages. Pulmonary arteries of ten lungs were injected by a colored mass of kemo-apoxy No. 151/20A, as well as $30 \%$ suspension of red lead oxide in kemo-apoxy No. 150 for cast formation and radiographic investigation respectively. For investigation of the bronchial artery, the other ten lungs were injected through the thoracic aorta with worm saline solution after ligation beyond the origin of the broncho-esophageal trunk. The injection used for radiograph comprised from $30 \%$ suspension of red lead oxide in $60 \%$ gum milk latex.

The present study had revealed that, the right pulmonary artery gave off; R. lobi cranialis, R. accessorius and R. lobi caudalis while the left one detached the R. lobi cranialis and R. lobi caudalis. Bronchovascular segmentation can be observed only in the accessory lobe and the caudal segment of the both caudal lobes, while the remaining of the lung was bronchoarterially segmented and the venous segmental rami were intersegmentally placed. The right bronchial artery gave off; R.bronchalis medius dexter, R.bronchalis accessorius and R.bronchalis caudalis dexter. While the left bronchial one detached; R.bronchalis cranialis sinister, R.esophagus, R.bronchalis medius sinister and R.bronchalis caudalis sinister.
\end{abstract}




\title{
The function and regulation of chick Ebf1 gene in somite development
}

\author{
Mohammed EL Sayed Rizk Abu EL Magd
}

Faculty of Veterinary Medicine, Kafr Al-Shaikh, Egypt

\begin{abstract}
The chick Ebf (Early B-cell Factor) gene is a member of a phylogenetically conserved EBF family of helix loop helix transcription factors. EBFs have been characterized for their roles in the differentiation of B-lymphocytes and subsequently implicated in various aspects of neural development. Recently, a new role for some EBFs in bone and muscle development has been investigated. However, their expression profile, regulation and function in the axial skeleton precursor, the somite, have yet to be elucidated. In this study, we have identified cEbf1 as a novel medial sclerotomal marker which is expressed under the control of Shh from the notochord. The organisation of cEbf1 expressing cells in the skeleton coupled with findings from tissue manipulation indicated that $c E b f 1$ exhibits a unique functional role related to skeletogenic cell specification, maturation and the differentiation of the epaxial myogenic cells. To identify the actual role of cEbf1 during somite differentiation, we have constructed a dominant negative form of this gene ( $\Delta \mathrm{Ebf} 1)$, which causes loss of Ebf1 function. Injection of $\Delta \mathrm{Ebf} 1$ into the developing somite resulted in down-regulation of both chondrogenic markers (Pax1 and Sox9) and myogenic marker (myoD) and up-regulation of the dermomyotomal marker (Pax3) a long with loss of vertebral body and pedicles and loss of epaxial muscles. This is the first study to demonstrate that $c E b f 1$ plays a critical role in the mediolateral patterning of somites and their skeletal and muscular derivatives. The other striking finding was the upregulation of some myogenic inhibitors such as myostatin after inhibition of cEbf1. This suggests that cEbf1 is necessary for muscle development and can increase muscle formation through inhibition of myostatin.
\end{abstract}




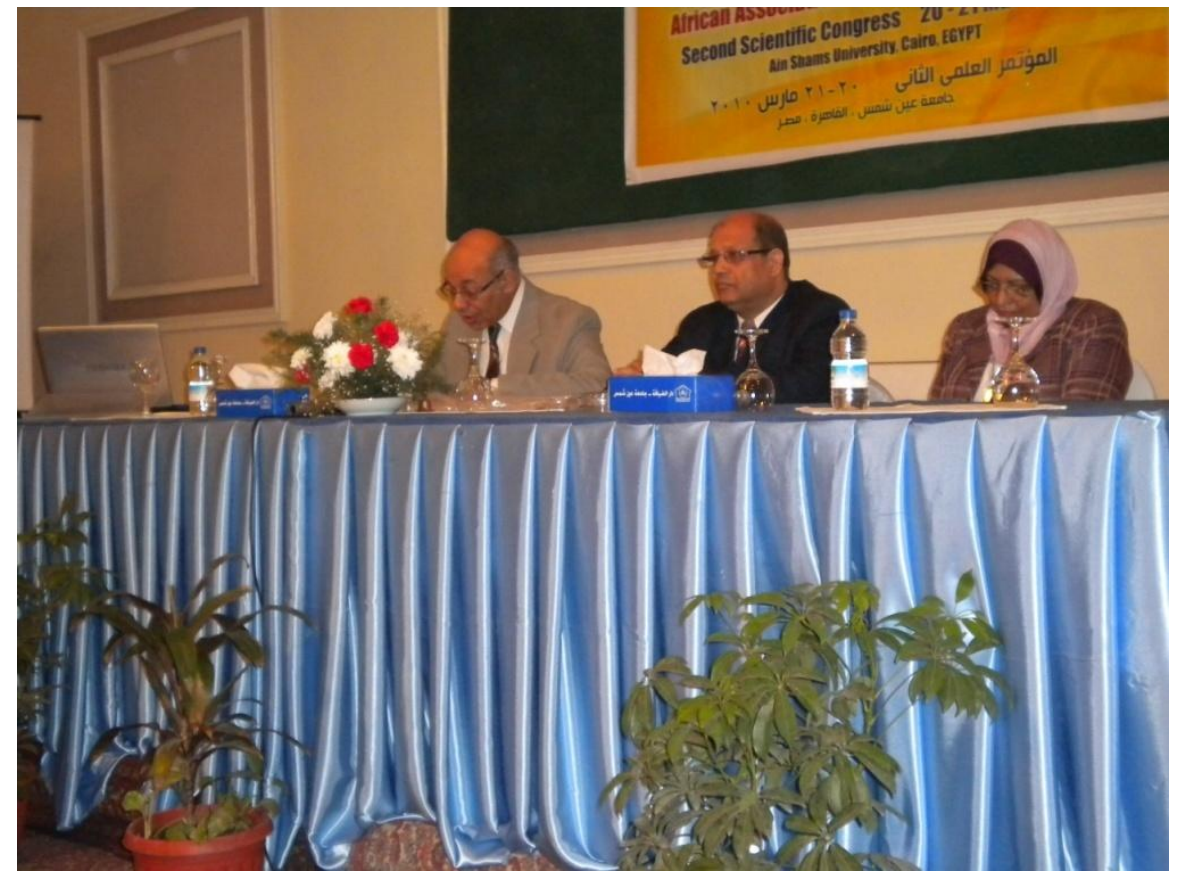

The opening speech presented by Prof. A. S. Saber, president of the Afr AVA (left). Prof. A. A. Mansour, the secretary (middle) and Prof. Sanna Al-Nahla, the treasurer (right)

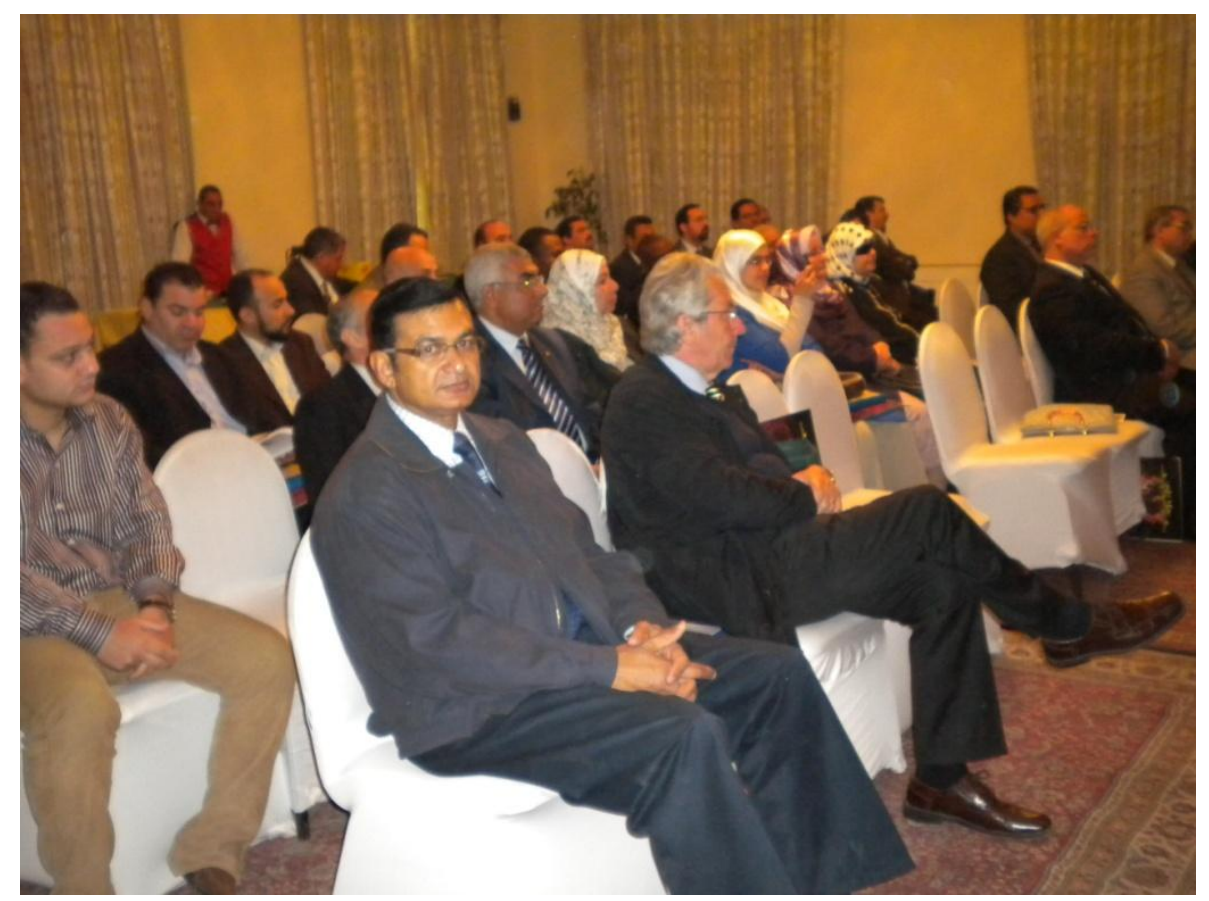

Attendees of the congress. Prof. Weyrauch (Germany) and Dr. Gahlot (India)

J. Vet. Anat.

Vol 3 No 1, (2010) 


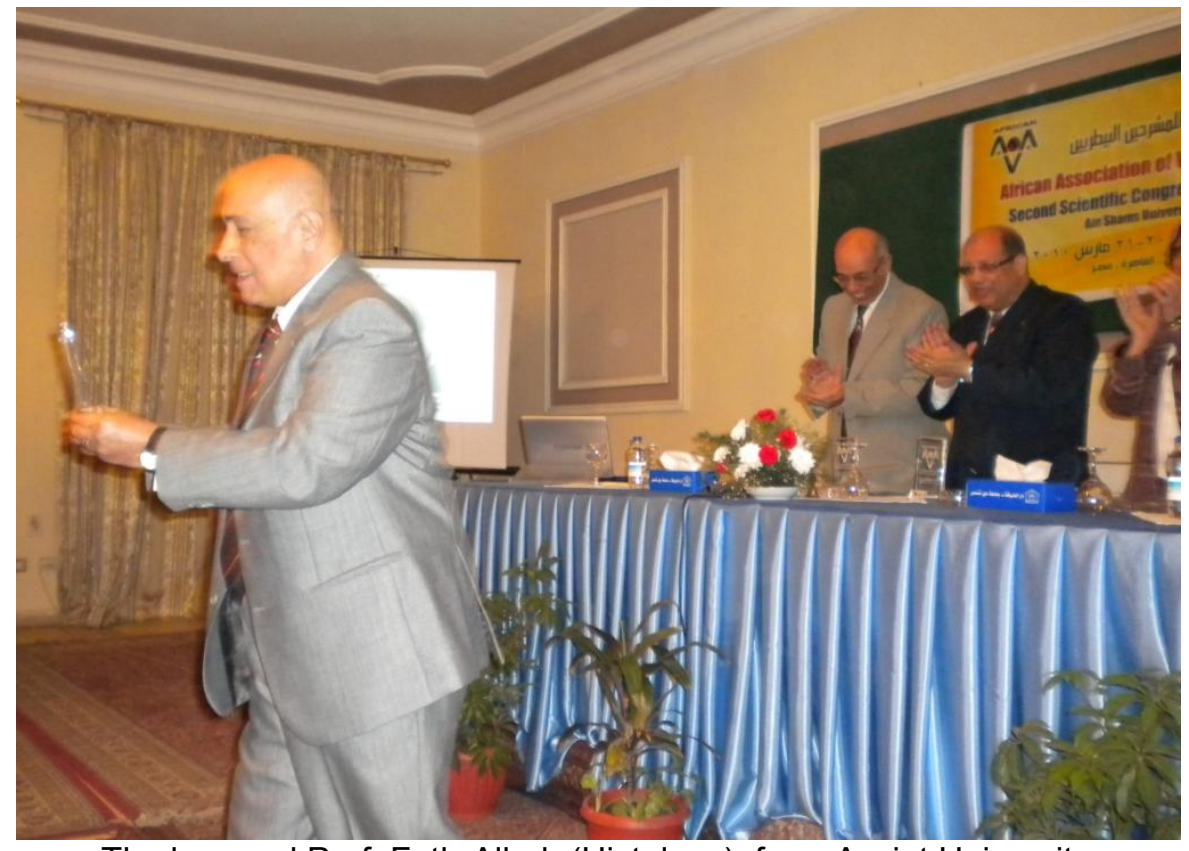

The honored Prof. Fath-Albab (Histology), from Assiut University

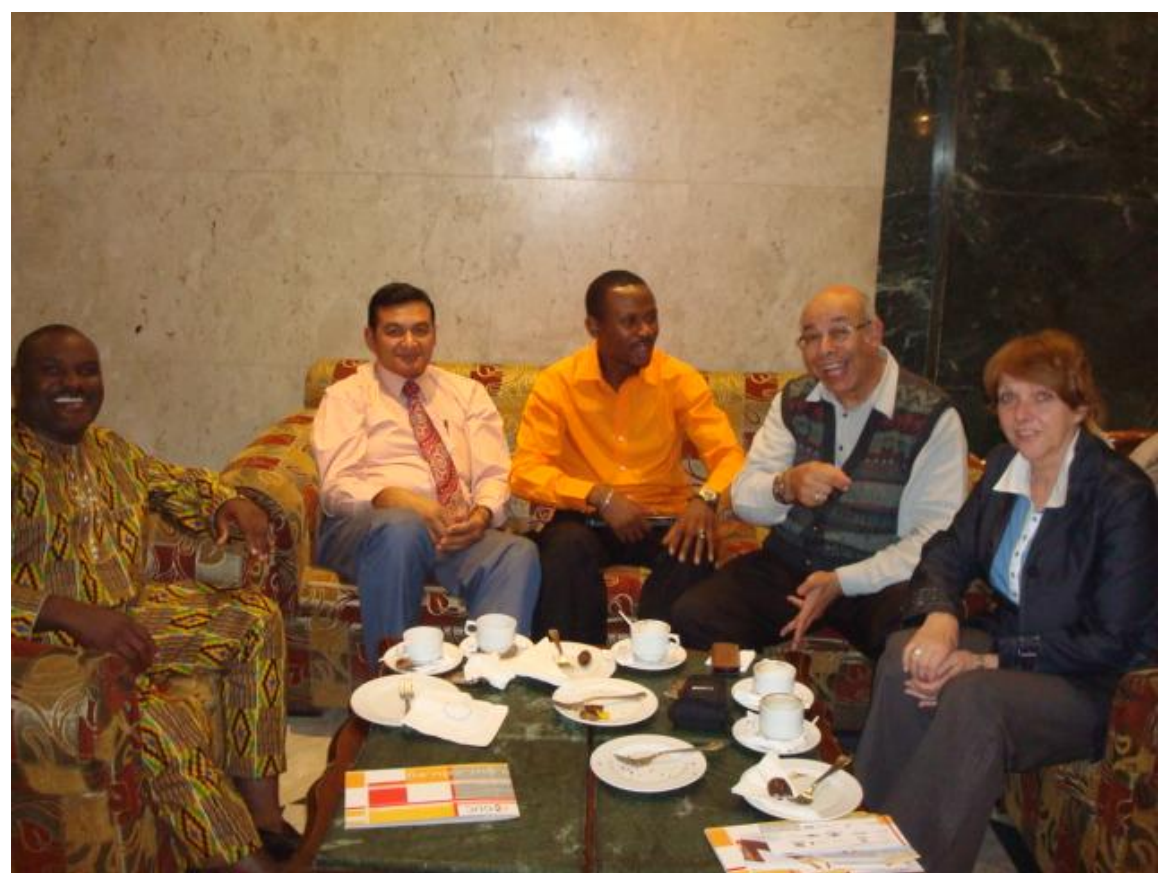

The Nigerian and Indian colleagues with Prof. Saber and Dr. Brigitte Schenk-Saber

J. Vet. Anat.

Vol 3 No 1, (2010) 